R.J. Hartmann, BSc, MSc, E. Lang, MD, T. Rich, MD, B. Ford, BPharm, PharmD, K. Lonergan, MSc, D. Wang, MSc, A. Mageau, BScN, MN, M. Kealey, BSc, MBA, M. Ejner, BSc, MBA, T. Junghans, BA; University of Calgary, Calgary, AB

Introduction: The addition of computerized physician order entry (CPOE) to Emergency Departments in recent years has led to speculation over potential benefits and pitfalls. Recent studies have shown benefits to CPOE, though there lacks sufficient evidence on how it could change physician behaviour. Physician practices are known to be difficult to change, with getting evidence into daily practice being the main challenge of knowledge translation. Our study aims were to determine if well-designed electronic order sets for CPOE improved MD practices. Methods: The Calgary Zone Pain Management in the Emergency Department Working Group relied on a GRADE-based literature review for identifying best practices for analgesia and antiemetics, resulting in soft changes to the dedicated analgesia and antiemetic electronic order set noting working group preference, and emphasizing hydromorphone over morphine, as well as $4 \mathrm{mg}$ ondansetron over $8 \mathrm{mg}$. The new electronic order set was started in the only Calgary Region order entry system on December 11th, 2014. Data was collected from July 2014 - May 2015. A Yates chi-squared analysis was completed on all orders in a category, as well as the subgroups of ED staff and residents, and orders placed using the new order set. Results: A total of 100460 orders were analyzed. The use of hydromorphone increased significantly across all 4 EDs. IV hydromorphone use increased $(5.82 \%$ of all opioid orders up to $26.93 \%, \mathrm{P}<0.0001)$ with a reciprocal decline in IV morphine (67.81\% of all opioid orders down to $46.56 \%, \mathrm{P}<0.0001)$. Similar effects were observed with ondansetron 4 mg IV orders increasing (1.37\% of all ondansetron orders to $18.64 \%$, $\mathrm{P}<0.0001)$ with a decrease in $8 \mathrm{mg}$ dosing $(15.75 \%$ of all ondansetron orders to $7.23 \%, \mathrm{P}<0.0001)$. These results were replicated to a lesser degree in the non-ED staff and non-order set subgroups. Implementation of the new order set resulted in an increase of its use $(37.64 \%$ of all opioid orders up to $49.29 \%, \mathrm{P}<0.0001)$. Finally, a cost-savings analysis was completed showing a projected annual savings of $\$ 185,676.52$ on medications alone. Conclusion: This data supports the manipulation of electronic order sets to help shape physician behaviour towards best practices. This provides another strong argument towards the benefits of CPOE, and can help maintain best practices in Emergency Medicine. Keywords: analgesia, electronic order sets, knowledge translation

\section{MP012 \\ Is there an association between the use of cardiac ultrasound and survival outcomes in patients arriving to the emergency department in cardiac arrest? The second Sonography in Hypotension and Cardiac Arrest in the Emergency Department (SHOC-ED 2) Study N. Beckett, BScH, P.R. Atkinson, MD, J. Fraser, BN, J. French, BSc, BM, Dip, IMC, RCS, Ed, D. Lewis, MBBS; Dalhousie Medicine New Brunswick, Saint John, NB}

Introduction: The use of cardiac point of care ultrasound (PoCUS) to assess cardiac arrest patients is widespread, although not mandated by advanced cardiac life support (ACLS) guidelines. This study aims to examine if the use of ultrasound, along with the findings on ultrasound are associated with a difference in outcomes of cardiac arrest patients in the emergency department (ED). Methods: A retrospective database and chart analysis was completed for patients arriving to a tertiary ED in asystole or PEA cardiac arrest, between 2010 and 2014. Patients were excluded if aged under 19, or with a previous DNR order. Patients were grouped based on whether PoCUS was used during ACLS (PoCUS group) and those without PoCUS (control group). Multiple data were abstracted from charts using a standardized form. Data was analyzed for the return of spontaneous circulation (ROSC), survival to hospital admission (SHA), and survival to hospital discharge (SHD), as well as initial cardiac activity findings on PoCUS. Results: 230 patients met the study inclusion criteria, with $44(19 \%)$ in the control group, and 186 $(81 \%)$ in the PoCUS group. In the PoCUS group 20 (11\%) had cardiac activity (Positive PoCUS) and $166(89 \%)$ had no cardiac activity recorded. The control group had a higher rate of SHA than the PoCUS group $(27 \%$; $95 \%$ CI $15-43 \%$ vs. $10 \%: 6-15 \%, \mathrm{p}=0.0046)$, however there was no difference in frequency of ROSC (control: $37 \%$; $24-55 \%$ vs. PoCUS 26\%; 20-33\%, p = 0.1373) or SHD (control: 7\%, 95\% CI 1-19\%; PoCUS: $2 \%, 95 \%$ CI 0-5\%, $\mathrm{p}=0.0858)$. Positive PoCUS patients had a higher frequency of ROSC $(75 \%$; $50-91 \%$ vs. $20 \%$; $15-$ $27 \%, \mathrm{p}<0.001)$ and SHA (25\%; $9-49 \%$ vs. $8 \% ; 4-13 \%, \mathrm{p}=0.0294)$ than patients with no PoCUS cardiac activity, however there was no difference in the rate of SHD between the positive PoCUS patients $(0 \%$; $0-17 \%)$ and patients with no PoCUS cardiac activity $(2 \% ; 0-5 \%, \mathrm{p}=$ 1.0000). Conclusion: Our results suggest that there is no difference in survival between cardiac arrest patients receiving PoCUS and those who do not. Although finding positive cardiac activity on PoCUS is associated with greater ROSC and survival to hospital admission, it does not identify patients with a final outcome of survival to hospital discharge. Keywords: point-of-care ultrasound (PoCUS), cardiac arrest, survival

\section{MP013}

A portrait of rural pre-hospital services in the province of Québec E. Bourdon, A.B. Tanguay, MD, MSc, F.K. Tounkara, A. Marois, R. Fleet, $\mathrm{MD}, \mathrm{PhD}$; Collège Ahuntsic, Département des soins préhospitaliers d'urgence, Montréal, QC

Introduction: Rural emergency departments (EDs) are important safety nets for $20 \%$ of Canadian citizens. In Quebec, the province's 26 rural EDs treat an average of 19,000 patients/year and are on average $300 \mathrm{~km}$ from levels 1 and 2 trauma centers. These distances signify that Emergency Medical Services (EMS) play a considerable role in the care of rural patients. EMS in Quebec province are private local services. There are no published reports on EMS in rural Quebec. As part of a larger study on rural emergency care, this descriptive study aimed at offering a comprehensive portrait of EMS. Methods: We conducted semi-structured interviews with managers of all paramedic services in rural Quebec. Interview questions focused on number of transports, training, availability of telemetry, GPS technologies, and work schedules. Results: Fifty managers of the 51 private companies serving the 26 rural EDs in Quebec were interviewed (response rate 98\%). All were primary care paramedics (PCP). In 2010, EMS transported 40,671 patients, with 10,228 emergency transports to the rural EDs. A total of 7956 inter-facility transfers were conducted, 1499 of them emergency. Each ED required between 88 and 700 inter-facility transfers. A total of $60 \%(\mathrm{n}=31 / 51)$ had GPS technology, only $25 \%(\mathrm{n}=13 / 51)$ had telemetry features. Work schedules varied with $13 \%(\mathrm{n}=7 / 51)$ of companies offering shifts of less than 12 hours, 28\% $(\mathrm{n}=14 / 51)$ 24/7 weekly shifts, and 56\% $(n=29 / 51)$ a combination. Conclusion: This is the first study to describe rural EMS in Quebec. The finding that Quebec's rural EDs are served by 51 privately-owned companies is unique in Canada. The considerable number of EMS transports, including inter-facility transfers, may reflect lack of local resources in rural EDs, the vulnerable population served, or the increased trauma risk in rural areas. Future studies should examine inter-facility transport reasons, costs, times and adequate training/scope of EMS practice.

Keywords: rural emergency departments, emergency medical services (EMS), transport 